\title{
A new iterative algorithm of pseudomonotone mappings for equilibrium problems in Hilbert spaces
}

Jong Kyu Kim* and Won Hee Lim

\section{"Correspondence:}

jongkyuk@kyungnam.ac.kr Department of Mathematics Education, Kyungnam University, Changwon, Gyeongnam 631-701 Korea

\begin{abstract}
In this paper, we introduce a new algorithm for finding a common element of the set of fixed points of $N$ strict pseudocontractions and the set of solutions of equilibrium problems with a pseudomonotone and Lipschitz-type continuous bifunction. The scheme is motivated by the idea of extragradient methods and fixed point iteration methods. We show that the iterative sequences generated by this algorithm converge strongly to the above mentioned common element under some suitable conditions on algorithm parameters in a real Hilbert space. And also, we consider the variational inequality problems as an application.
\end{abstract}

MSC: 46H09; 47H10;47J25; 65K10

Keywords: strict pseudocontractions; pseudomonotone; Lipschitz-type continuous; equilibrium problems; fixed points

\section{Introduction}

Let $C$ be a nonempty closed convex subset of a real Hilbert space $H$ with the inner product $\langle\cdot, \cdot\rangle$ and the norm $\|\cdot\|$, and let $f$ be a bifunction from $C \times C$ into $\mathbf{R}$ such that $f(x, x)=0$ for all $x \in C$. We consider the equilibrium problem in the sense of Blum and Oettli [1]: Find $x^{\prime \prime} \in C$ such that

$$
f\left(x^{*}, y\right) \geq 0
$$

for all $y \in C$.

We denote by $\operatorname{Sol}(E P(f))$ the set of solutions of the equilibrium problem $E P(f)$.

We know that the problem $E P(f, C)$ covers many important problems in optimization and nonlinear analysis. It has also found many applications in economics, transportation and engineering (see $[1,2]$ and the references quoted therein). Theory and methods for solving this problem have been developed by many authors [3-7]. Alternatively, the problem of finding a common fixed point of a sequence of finite self-mappings $\left\{S_{i}\right\}_{i=1}^{N}(N \geq 1)$ is described as follows: Find $x^{*} \in C$ such that

$$
x^{*} \in \bigcap_{i=1}^{N} F\left(S_{i}\right),
$$

\section{Springer}

(C) 2013 Kim and Lim; licensee Springer. This is an Open Access article distributed under the terms of the Creative Commons Attribution License (http://creativecommons.org/licenses/by/2.0), which permits unrestricted use, distribution, and reproduction in any medium, provided the original work is properly cited. 
where $F\left(S_{i}\right)$ is the set of fixed points of the mappings $S_{i}(i=1, \ldots, N)$ on $C$. This problem has now become a mature subject in nonlinear analysis. The theory and solution methods of this problem can be found in many research papers and monographs (see [8-10]).

We are interested in the problem of finding a common element of the set of solutions of the equilibrium problem $E P(f)$ and the set of solutions of the fixed problem $(F P)$, namely: Find $x^{*} \in C$ such that

$$
x^{*} \in \bigcap_{i=1}^{N} F\left(S_{i}\right) \cap \operatorname{Sol}(E P(f)) .
$$

A special case of problem (1.1) is that $f(x, y)=\langle F(x), y-x\rangle$, and this problem is reduced to finding a common element of the set of solutions of variational inequalities, i.e., find $x^{*} \in C$ such that

$$
\left\langle F\left(x^{*}\right), x-x^{*}\right\rangle \geq 0, \quad \forall x \in C,
$$

and the set solutions of a fixed point problem (see [11-17]).

In this paper, we introduce a new iterative scheme for solving problem (1.1). This method can be considered to be an improvement of the viscosity approximation method in $[15,18$, $19]$ and the iterative method in [20] via an improvement of the extragradient methods [3, $4,21-23]$.

The paper is organized as follows. Section 2 recalls some concepts in equilibrium problems and fixed point problems that are used in the sequel and an iterative algorithm for solving problem (1.1). In Section 3, we prove the convergence theorems for the algorithms which are defined in Section 2 as the main results of this paper. In Section 4, we consider the variational inequality problems as an application of the main theorem.

\section{Preliminaries}

We first recall the following definitions that will be used for the main theorem.

Definition 2.1 Let $C$ be a nonempty closed convex subset of a real Hilbert space $H$. A bifunction $f: C \times C \rightarrow \mathbf{R}$ is said to be

(a) monotone on $C$ if $f(x, y)+f(y, x) \leq 0, \forall x, y \in C$;

(b) pseudomonotone on $C$ if $f(x, y) \geq 0$ implies $f(y, x) \leq 0, \forall x, y \in C$;

(c) Lipschitz-type continuous on $C$ with two constants $c_{1}>0$ and $c_{2}>0$ if

$$
f(x, y)+f(y, z) \geq f(x, z)-c_{1}\|x-y\|^{2}-c_{2}\|y-z\|^{2}, \quad \forall x, y, z \in C .
$$

We know that every monotone bifunction $f$ is pseudomonotone, but the converse is not true (see [24]).

Definition 2.2 Let $C$ be a nonempty closed convex subset of a real Hilbert space $H$. A mapping $S: C \rightarrow C$ is said to be a strict pseudocontraction if there exists a constant $0 \leq L<1$ such that

$$
\|S(x)-S(y)\|^{2} \leq\|x-y\|^{2}+L\|(I-S)(x)-(I-S)(y)\|^{2}, \quad \forall x, y \in C,
$$

where $I$ is the identity mapping on $H$. If $L=0$, then $S$ is called nonexpansive on $C$. 
Now, we define the projection on $C$, denoted by $\operatorname{Pr}_{C}(\cdot)$, i.e.,

$$
\operatorname{Pr}_{C}(x)=\operatorname{argmin}\{\|y-x\|: y \in C\}, \quad \forall x \in H
$$

And we use the symbols $\rightarrow$ and $\rightarrow$ to denote weak convergence and strong convergence, respectively. The following proposition gives some useful properties for strict pseudocontractions.

Proposition 2.3 [25] Let $C$ be a nonempty closed convex subset of a real Hilbert space $H$, let $S: C \rightarrow C$ be an L-strict pseudocontraction, and for each $i=1, \ldots, N$, let $S_{i}: C \rightarrow C$ be an $L_{i}$-strict pseudocontraction for some $0 \leq L_{i}<1$. Then we have the following.

(a) S satisfies the following Lipschitz condition:

$$
\|S(x)-S(y)\| \leq \frac{1+L}{1-L}\|x-y\|, \quad \forall x, y \in C
$$

(b) $(I-S)$ is demiclosed at zero. That is, if the sequence $\left\{x^{k}\right\}$ is in $C$ such that $x^{k} \rightarrow \bar{x}$ and $(I-S)\left(x^{k}\right) \rightarrow 0$, then $(I-S)(\bar{x})=0$

(c) The set $F(S)$ is closed and convex;

(d) If $\lambda_{i}>0(i=1, \ldots, N)$ and $\sum_{i=1}^{N} \lambda_{i}=1$, then $\sum_{i=1}^{N} \lambda_{i} S_{i}$ is an $\bar{L}$-strict pseudocontraction, where $\bar{L}:=\max \left\{L_{i} \mid 1 \leq i \leq N\right\}$;

(e) If $\lambda_{i}$ is the same as in (d) and $\left\{S_{i} \mid i=1, \ldots, N\right\}$ has a common fixed point, then

$$
F\left(\sum_{i=1}^{N} \lambda_{i} S_{i}\right)=\bigcap_{i=1}^{N} F\left(S_{i}\right) .
$$

Many authors studied the problem of finding a common fixed point of a finite family of mappings. For instance, Marino and Xu [26] constructed an iterative algorithm for finding a common fixed point of $N$ strict pseudocontractions $S_{i}(i=1, \ldots, N)$. They defined the sequence $\left\{x^{k}\right\}$ starting from $x^{0} \in H$ and taking

$$
x^{k+1}=\alpha_{k} x^{k}+\left(1-\alpha_{k}\right) \sum_{i=1}^{N} \lambda_{k, i} S_{i}\left(x^{k}\right)
$$

where the control sequence of parameters $\left\{\lambda_{k}\right\}$ was made in order to get the guarantee for the convergence of the iterative sequence $\left\{x^{k}\right\}$. And they proved that the sequence $\left\{x^{k}\right\}$ converges weakly to the point $\bar{x} \in \bigcap_{i=1}^{N} F\left(S_{i}\right)$.

Recently, Chen et al. [20] introduced a new iterative scheme for finding a common element of the set of common fixed points of a sequence of strict pseudocontractions $\left\{\bar{S}_{i}\right\}$ and the set of solutions of the equilibrium problem $E P(f)$ in a real Hilbert space $H$. Given a starting point $x^{0} \in H$, three iterative sequences $\left\{x^{k}\right\},\left\{y^{k}\right\}$ and $\left\{z^{k}\right\}$ are generated as the following scheme:

$$
\left\{\begin{array}{l}
\text { Compute } y^{k}=\alpha_{k} x^{k}+\left(1-\alpha_{k}\right) \bar{S}_{k}\left(x^{k}\right) ; \\
\text { Find } z^{k} \in C \text { such that } f\left(z^{k}, y\right)+\frac{1}{r_{k}}\left\langle y-z^{k}, z^{k}-y^{k}\right\rangle \geq 0, \forall y \in C ; \\
\text { Compute } x^{k+1}=\operatorname{Pr}_{C_{k}}\left(x^{0}\right) \text {, where } C_{k}:=\left\{v \in C \mid\left\|z^{k}-v\right\| \leq\left\|x^{k}-v\right\|\right\} .
\end{array}\right.
$$


Here, two sequences $\left\{\alpha_{k}\right\}$ and $\left\{r_{k}\right\}$ are given as control parameters. The authors proved that the sequences $\left\{x^{k}\right\},\left\{y^{k}\right\}$ and $\left\{z^{k}\right\}$ converged strongly to the same point $x^{*}$, under certain conditions on $\left\{\alpha_{k}\right\}$ and $\left\{r_{k}\right\}$, such that

$$
x^{*} \in \operatorname{Pr}_{\operatorname{Sol}(E P(f)) \cap F(S)}\left(x^{0}\right) \text {, }
$$

where $S$ is a nonexpansive mapping of $C$ into itself defined by

$$
S(x)=\lim _{j \rightarrow \infty} \bar{S}_{j}(x)
$$

for all $x \in C$.

The methods for finding a common element of the sets $\operatorname{Sol}(E P(f))$ and $\bigcap_{i=1}^{N} F\left(S_{i}\right)$ in a real Hilbert space have been studied in many research papers (see [7, 17, 21, 22, 27-30]).

We need the following assumptions for the main theorems.

Assumption 2.4 The bifunction $f$ satisfies the following conditions:

(i) $f$ is pseudomonotone and weakly continuous on $C$;

(ii) $f$ is Lipschitz-type continuous on $C$;

(iii) for each $x \in C, f(x, \cdot)$ is convex and subdifferentiable on $C$.

Assumption 2.5 Every $S_{i}$ is an $L_{i}$-strict pseudocontraction for some $0 \leq L_{i}<1$.

Assumption 2.6 The solution set of (1.1) is nonempty, i.e.,

$$
\bigcap_{i=1}^{N} F\left(S_{i}\right) \cap \operatorname{Sol}(E P(f)) \neq \emptyset .
$$

Note that if $C \subseteq \operatorname{ri}(\operatorname{dom}(f(x, \cdot)))$, where $\operatorname{ri}(\operatorname{dom}(f(x, \cdot)))$ is the set of relative interior points of the domain of $f(x, \cdot)$, then Assumption 2.4(iii) is satisfied. Now we construct the new algorithms as follows.

\section{Algorithm 2.7}

Initialization: Choose positive sequences $\left\{\lambda_{k}\right\},\left\{\alpha_{k}\right\},\left\{\beta_{k}\right\},\left\{\gamma_{k}\right\}$ and $\left\{\lambda_{k, i}\right\}$ satisfying the following conditions:

$$
\left\{\begin{array}{l}
\alpha_{k}+\beta_{k} \leq 1, \quad \forall k \geq 0, \\
\liminf _{k \rightarrow \infty} \beta_{k} \in(0,1), \\
\liminf _{k \rightarrow \infty} \frac{\alpha_{k}}{\alpha_{k}+\beta_{k}} \in(\bar{L}, 1), \quad \text { where } \bar{L}:=\max \left\{L_{i} \mid 1 \leq i \leq N\right\}, \\
\liminf _{k \rightarrow \infty}\left(\gamma_{k}+\left(1-\gamma_{k}\right)\left(\alpha_{k}+\beta_{k}\right)\right)>0, \quad\left\{\gamma_{k}\right\} \subset(0,1), \\
\left\{\lambda_{k}\right\} \subset[a, b] \quad \text { for some } a, b \in\left(0, \frac{1}{L}\right), \text { where } L:=\max \left\{2 c_{1}, 2 c_{2}\right\}, \\
\sum_{i=1}^{p} \lambda_{k, i}=1 \quad \text { for all } k \geq 1 .
\end{array}\right.
$$

Take an initial point $x^{0} \in C$ and set $k:=0$.

Iteration $k$ : Carry out three steps below continuously. 
- Step 1. Solve two strongly convex programs:

$$
\left\{\begin{array}{l}
y^{k}:=\operatorname{argmin}\left\{\lambda_{k} f\left(x^{k}, y\right)+\frac{1}{2}\left\|y-x^{k}\right\|^{2} \mid y \in C\right\}, \\
t^{k}:=\operatorname{argmin}\left\{\lambda_{k} f\left(y^{k}, y\right)+\frac{1}{2}\left\|y-x^{k}\right\|^{2} \mid y \in C\right\} .
\end{array}\right.
$$

- Step 2. Compute the iterations

$$
\left\{\begin{array}{l}
\bar{y}^{k}:=\left(1-\gamma_{k}\right) x^{k}+\gamma_{k} t^{k} \\
z^{k}:=\left(1-\alpha_{k}-\beta_{k}\right) \bar{y}^{k}+\alpha_{k} t^{k}+\beta_{k} \sum_{i=1}^{N} \lambda_{k, i} S_{i}\left(t^{k}\right)
\end{array}\right.
$$

- Step 3. Set

$$
\left\{\begin{aligned}
C_{k}:=\left\{z \in C \mid\left\|z^{k}-z\right\|^{2} \leq\left\|x^{k}-z\right\|^{2}-\beta_{k}\left(\frac{\alpha_{k}}{\alpha_{k}+\beta_{k}}-\bar{L}\right)\left\|\bar{S}_{k}\left(t^{k}\right)-t^{k}\right\|^{2}\right\}, \\
\quad \text { where } \bar{S}_{k}:=\sum_{i=1}^{N} \lambda_{k, i} S_{i}\left(x^{k}\right), \\
Q_{k}:=\left\{z \in C \mid\left\langle x^{k}-z, x^{0}-x^{k}\right\rangle \geq 0\right\} .
\end{aligned}\right.
$$

Compute $x^{k+1}:=\operatorname{Pr}_{C_{k} \cap Q_{k}}\left(x^{0}\right)$.

Increase $k$ by one and go back to Step 1 .

\section{Convergence of the algorithms}

In this section, we study the convergence of Algorithm 2.7. We need the following useful lemmas for the main theorems.

Lemma 3.1 [2] Let $C$ be a nonempty closed convex subset of a real Hilbert space $H$, and let $g: C \rightarrow \mathbf{R}$ be subdifferentiable on $C$. Then $x^{\prime \prime}$ is a solution of the following convex problem:

$$
\min \{g(x) \mid x \in C\}
$$

if and only if

$$
0 \in \partial g\left(x^{*}\right)+N_{C}\left(x^{*}\right)
$$

where $\partial g(\cdot)$ denotes the subdifferential of $g$ and $N_{C}\left(x^{*}\right)$ is the (outward) normal cone of $C$ at $x^{\prime \prime} \in C$.

Lemma 3.2 [8] Let $C$ be a nonempty closed convex subset of a real Hilbert space $H$ and $x^{0} \in H$. Let $\left\{x^{k}\right\}$ be a bounded sequence such that every weakly cluster point $\bar{x}$ of $\left\{x^{k}\right\}$ belongs to $C$ and

$$
\left\|x^{k}-x^{0}\right\| \leq\left\|x^{0}-\operatorname{Pr}_{C}\left(x^{0}\right)\right\|, \quad \forall k \geq 0 .
$$

Then $\left\{x^{k}\right\}$ converges strongly to $\operatorname{Pr}_{C}\left(x^{0}\right)$ as $k \rightarrow \infty$.

Now, we are in a position to prove the main theorem. 
Theorem 3.3 Let C be a nonempty closed convex subset of a real Hilbert space H. Suppose that Assumptions 2.4-2.6 are satisfied. Then the sequences $\left\{x^{k}\right\},\left\{y^{k}\right\}$ and $\left\{z^{k}\right\}$ generated by Algorithm 2.7 converge strongly to the same point $x^{*} \in \bigcap_{i=1}^{N} F\left(S_{i}\right) \cap \operatorname{Sol}(E P(f))$, where

$$
x^{*}=\operatorname{Pr}_{\bigcap_{i=1}^{N} F\left(S_{i}\right) \cap \operatorname{Sol}(E P(f))}\left(x^{0}\right) .
$$

Proof The proof of this theorem is divided into several steps.

Step 1. Suppose that $x^{\prime \prime} \in \bigcap_{i=1}^{N} F\left(S_{i}\right) \cap \operatorname{Sol}(E P(f))$. Then we have

$$
\begin{aligned}
\left\|t^{k}-x^{*}\right\|^{2} \leq & \left\|x^{k}-x^{*}\right\|^{2}-\left(1-2 \lambda_{k} c_{2}\right)\left\|t^{k}-y^{k}\right\|^{2} \\
& -\left(1-2 \lambda_{k} c_{1}\right)\left\|x^{k}-y^{k}\right\|^{2}, \quad \forall k \geq 0 .
\end{aligned}
$$

Since $f(x, \cdot)$ is convex on $C$ for each $x \in C$, by Lemma 3.1, we see that

$$
t^{k}=\operatorname{argmin}\left\{\frac{1}{2}\left\|t-x^{k}\right\|^{2}+\lambda_{k} f\left(y^{k}, t\right) \mid t \in C\right\}
$$

if and only if

$$
0 \in \partial_{2}\left(\lambda_{k} f\left(y^{k}, y\right)+\frac{1}{2}\left\|y-x^{k}\right\|^{2}\right)\left(t^{k}\right)+N_{C}\left(t^{k}\right)
$$

where $N_{C}(x)$ is the (outward) normal cone of $C$ at $x \in C$.

Since $f\left(y^{k}, \cdot\right)$ is subdifferentiable on $C$, by the well-known Moreau-Rockafellar theorem (see [31]), there exists $w \in \partial_{2} f\left(y^{k}, t^{k}\right)$ such that

$$
f\left(y^{k}, t\right)-f\left(y^{k}, t^{k}\right) \geq\left\langle w, t-t^{k}\right\rangle, \quad \forall t \in C .
$$

Substituting $t=x^{*}$ into this inequality, we obtain

$$
f\left(y^{k}, x^{*}\right)-f\left(y^{k}, t^{k}\right) \geq\left\langle w, x^{*}-t^{k}\right\rangle .
$$

And also, it follows from (3.3) that $0=\lambda_{k} w+t^{k}-x^{k}+\bar{w}$, where $w \in \partial_{2} f\left(y^{k}, t^{k}\right)$ and $\bar{w} \in$ $N_{C}\left(t^{k}\right)$. By the definition of the normal cone $N_{C}$, we have

$$
\left\langle t^{k}-x^{k}, t-t^{k}\right\rangle \geq \lambda_{k}\left\langle w, t^{k}-t\right\rangle, \quad \forall t \in C .
$$

Substituting $t=x^{*} \in C$ into the last inequality, we obtain

$$
\left\langle t^{k}-x^{k}, x^{*}-t^{k}\right\rangle \geq \lambda_{k}\left\langle w, t^{k}-x^{*}\right\rangle .
$$

Combining (3.4) and (3.6), we have

$$
\left\langle t^{k}-x^{k}, x^{*}-t^{k}\right\rangle \geq \lambda_{k}\left(f\left(y^{k}, t^{k}\right)-f\left(y^{k}, x^{*}\right)\right) .
$$

Since $x^{*} \in \operatorname{Sol}(E P(f)), f\left(x^{*}, y\right) \geq 0$ for all $y \in C$, and $f$ is pseudomonotone on $C$, we have $f\left(y^{k}, x^{*}\right) \leq 0$. Hence, (3.7) implies that

$$
\left\langle t^{k}-x^{k}, x^{*}-t^{k}\right\rangle \geq \lambda_{k} f\left(y^{k}, t^{k}\right) .
$$


From Lipschitz condition (2.1) for $f$ with $x=x^{k}, y=y^{k}$ and $z=t^{k}$, we have

$$
f\left(y^{k}, t^{k}\right) \geq f\left(x^{k}, t^{k}\right)-f\left(x^{k}, y^{k}\right)-c_{1}\left\|y^{k}-x^{k}\right\|^{2}-c_{2}\left\|t^{k}-y^{k}\right\|^{2} .
$$

Combining (3.8) and (3.9), we get

$$
\left\langle t^{k}-x^{k}, x^{*}-t^{k}\right\rangle \geq \lambda_{k}\left(f\left(x^{k}, t^{k}\right)-f\left(x^{k}, y^{k}\right)-c_{1}\left\|y^{k}-x^{k}\right\|^{2}-c_{2}\left\|t^{k}-y^{k}\right\|^{2}\right) .
$$

Similarly, since $y^{k}$ is the unique solution of the strongly convex program

$$
\min \left\{\frac{1}{2}\left\|y-x^{k}\right\|^{2}+\lambda_{k} f\left(x^{k}, y\right) \mid y \in C\right\}
$$

we have

$$
\lambda_{k}\left(f\left(x^{k}, y\right)-f\left(x^{k}, y^{k}\right)\right) \geq\left\langle y^{k}-x^{k}, y^{k}-y\right\rangle, \quad \forall y \in C .
$$

Substituting $y=t^{k} \in C$ into the last inequality, we have

$$
\lambda_{k}\left(f\left(x^{k}, t^{k}\right)-f\left(x^{k}, y^{k}\right)\right) \geq\left\langle y^{k}-x^{k}, y^{k}-t^{k}\right\rangle .
$$

Since

$$
2\left\langle t^{k}-x^{k}, x^{*}-t^{k}\right\rangle=\left\|x^{k}-x^{*}\right\|^{2}-\left\|t^{k}-x^{k}\right\|^{2}-\left\|t^{k}-x^{*}\right\|^{2},
$$

from (3.10), (3.11), we have

$$
\begin{gathered}
\left\|x^{k}-x^{*}\right\|^{2}-\left\|t^{k}-x^{k}\right\|^{2}-\left\|t^{k}-x^{*}\right\|^{2} \geq \\
2\left\langle y^{k}-x^{k}, y^{k}-t^{k}\right\rangle-2 \lambda_{k} c_{1}\left\|x^{k}-y^{k}\right\|^{2} \\
-2 \lambda_{k} c_{2}\left\|t^{k}-y^{k}\right\|^{2} .
\end{gathered}
$$

Hence, we have

$$
\begin{aligned}
\left\|t^{k}-x^{*}\right\|^{2} \leq & \left\|x^{k}-x^{*}\right\|^{2}-\left\|t^{k}-x^{k}\right\|^{2}-2\left\langle y^{k}-x^{k}, y^{k}-t^{k}\right\rangle \\
& +2 \lambda_{k} c_{1}\left\|x^{k}-y^{k}\right\|^{2}+2 \lambda_{k} c_{2}\left\|t^{k}-y^{k}\right\|^{2} \\
= & \left\|x^{k}-x^{*}\right\|^{2}-\left\|\left(t^{k}-y^{k}\right)+\left(y^{k}-x^{k}\right)\right\|^{2}-2\left\langle y^{k}-x^{k}, y^{k}-t^{k}\right\rangle \\
& +2 \lambda_{k} c_{1}\left\|x^{k}-y^{k}\right\|^{2}+2 \lambda_{k} c_{2}\left\|t^{k}-y^{k}\right\|^{2} \\
\leq & \left\|x^{k}-x^{*}\right\|^{2}-\left\|t^{k}-y^{k}\right\|^{2}-\left\|x^{k}-y^{k}\right\|^{2}+2 \lambda_{k} c_{1}\left\|x^{k}-y^{k}\right\|^{2}+2 \lambda_{k} c_{2}\left\|t^{k}-y^{k}\right\|^{2} \\
= & \left\|x^{k}-x^{*}\right\|^{2}-\left(1-2 \lambda_{k} c_{1}\right)\left\|x^{k}-y^{k}\right\|^{2}-\left(1-2 \lambda_{k} c_{2}\right)\left\|y^{k}-t^{k}\right\|^{2} .
\end{aligned}
$$

The implies that the inequality (3.2) holds.

Step 2. Next, we show that

$$
\bigcap_{i=1}^{N} F\left(S_{i}\right) \cap \operatorname{Sol}(E P(f)) \subseteq C_{k}
$$

for all $k \geq 0$. 
Using Step 1 and $\bar{y}^{k}=\left(1-\gamma_{k}\right) x^{k}+\gamma_{k} t^{k}$, we have

$$
\begin{aligned}
\left\|\bar{y}^{k}-x^{*}\right\|^{2}= & \left\|\left(1-\gamma_{k}\right)\left(x^{k}-x^{*}\right)+\gamma_{k}\left(t^{k}-x^{*}\right)\right\|^{2} \\
\leq & \left(1-\gamma_{k}\right)\left\|x^{k}-x^{*}\right\|^{2}+\gamma_{k}\left\|t^{k}-x^{*}\right\|^{2} \\
\leq & \left(1-\gamma_{k}\right)\left\|x^{k}-x^{*}\right\|^{2}+\gamma_{k}\left\{\left\|x^{k}-x^{*}\right\|^{2}-\left(1-2 \lambda_{k} c_{1}\right)\left\|x^{k}-y^{k}\right\|^{2}\right. \\
& \left.-\left(1-2 \lambda_{k} c_{2}\right)\left\|y^{k}-t^{k}\right\|^{2}\right\} \\
= & \left\|x^{k}-x^{*}\right\|^{2}-\gamma_{k}\left(1-2 \lambda_{k} c_{1}\right)\left\|x^{k}-y^{k}\right\|^{2}-\gamma_{k}\left(1-2 \lambda_{k} c_{2}\right)\left\|y^{k}-t^{k}\right\|^{2},
\end{aligned}
$$

where $x^{*} \in \operatorname{Sol}(E P(f))$.

Set

$$
\bar{S}_{k}:=\sum_{i=1}^{N} \lambda_{k, i} S_{i} .
$$

Let $x^{*} \in \bigcap_{i=1}^{p} \operatorname{Fix}\left(S_{i}\right) \cap \operatorname{Sol}(E P(f))$, using Proposition 2.3(d), (3.12) and the relation

$$
\|\lambda x+(1-\lambda) y\|^{2}=\lambda\|x\|^{2}+(1-\lambda)\|y\|^{2}-\lambda(1-\lambda)\|x-y\|^{2}, \quad \forall x, y \in H, \lambda \in[0,1],
$$

and $z^{k}=\left(1-\alpha_{k}-\beta_{k}\right) \bar{y}^{k}+\alpha_{k} t^{k}+\beta_{k} \bar{S}_{k}\left(t^{k}\right)$, we have

$$
\begin{aligned}
& \left\|z^{k}-x^{*}\right\|^{2} \\
& =\left\|\left(1-\alpha_{k}-\beta_{k}\right)\left(\bar{y}^{k}-x^{*}\right)+\left(\alpha_{k}+\beta_{k}\right) \frac{1}{\alpha_{k}+\beta_{k}}\left\{\alpha_{k}\left(t^{k}-x^{*}\right)+\beta_{k}\left(\bar{S}_{k}\left(t^{k}\right)-x^{*}\right)\right\}\right\|^{2} \\
& \leq\left(1-\alpha_{k}-\beta_{k}\right)\left\|\bar{y}^{k}-x^{*}\right\|^{2}+\left(\alpha_{k}+\beta_{k}\right)\left\|\frac{\alpha_{k}}{\alpha_{k}+\beta_{k}}\left(t^{k}-x^{*}\right)+\frac{\beta_{k}}{\alpha_{k}+\beta_{k}}\left(\bar{S}_{k}\left(t^{k}\right)-x^{*}\right)\right\|^{2} \\
& =\left(1-\alpha_{k}-\beta_{k}\right)\left\|\bar{y}^{k}-x^{*}\right\|^{2}+\alpha_{k}\left\|t^{k}-x^{*}\right\|^{2}+\beta_{k}\left\|\bar{S}_{k}\left(t^{k}\right)-x^{*}\right\|^{2}-\frac{\alpha_{k} \beta_{k}}{\alpha_{k}+\beta_{k}}\left\|\bar{S}_{k}\left(t^{k}\right)-t^{k}\right\|^{2} \\
& \leq\left(1-\alpha_{k}-\beta_{k}\right)\left\|\bar{y}^{k}-x^{*}\right\|^{2}+\alpha_{k}\left\|t^{k}-x^{*}\right\|^{2} \\
& +\beta_{k}\left(\left\|t^{k}-x^{*}\right\|^{2}+\bar{L}\left\|\left(I-\bar{S}_{k}\right)\left(t^{k}\right)-\left(I-\bar{S}_{k}\right)\left(x^{*}\right)\right\|^{2}\right)-\frac{\alpha_{k} \beta_{k}}{\alpha_{k}+\beta_{k}}\left\|\bar{S}_{k}\left(t^{k}\right)-t^{k}\right\|^{2} \\
& \leq\left(1-\alpha_{k}-\beta_{k}\right)\left\|\bar{y}^{k}-x^{*}\right\|^{2}+\left(\alpha_{k}+\beta_{k}\right)\left\|t^{k}-x^{*}\right\|^{2}+\left(\beta_{k} \bar{L}-\frac{\alpha_{k} \beta_{k}}{\alpha_{k}+\beta_{k}}\right)\left\|\bar{S}_{k}\left(t^{k}\right)-t^{k}\right\|^{2} \\
& \leq\left(1-\alpha_{k}-\beta_{k}\right)\left(\left\|x^{k}-x^{*}\right\|^{2}-\gamma_{k}\left(1-2 \lambda_{k} c_{1}\right)\left\|x^{k}-y^{k}\right\|^{2}-\gamma_{k}\left(1-2 \lambda_{k} c_{2}\right)\left\|y^{k}-t^{k}\right\|^{2}\right) \\
& +\left(\alpha_{k}+\beta_{k}\right)\left(\left\|x^{k}-x^{*}\right\|^{2}-\left(1-2 \lambda_{k} c_{1}\right)\left\|x^{k}-y^{k}\right\|^{2}-\left(1-2 \lambda_{k} c_{2}\right)\left\|y^{k}-t^{k}\right\|^{2}\right) \\
& +\left(\beta_{k} \bar{L}-\frac{\alpha_{k} \beta_{k}}{\alpha_{k}+\beta_{k}}\right)\left\|\bar{S}_{k}\left(t^{k}\right)-t^{k}\right\|^{2} \\
& \leq\left\|x^{k}-x^{*}\right\|^{2}-m_{k}\left(1-2 \lambda_{k} c_{1}\right)\left\|x^{k}-y^{k}\right\|^{2}-m_{k}\left(1-2 \lambda_{k} c_{2}\right)\left\|y^{k}-t^{k}\right\|^{2} \\
& -\beta_{k}\left(\frac{\alpha_{k}}{\alpha_{k}+\beta_{k}}-\bar{L}\right)\left\|\bar{S}_{k}\left(t^{k}\right)-t^{k}\right\|^{2} \\
& \leq\left\|x^{k}-x^{*}\right\|^{2}-\beta_{k}\left(\frac{\alpha_{k}}{\alpha_{k}+\beta_{k}}-\bar{L}\right)\left\|\bar{S}_{k}\left(t^{k}\right)-t^{k}\right\|^{2} \text {, }
\end{aligned}
$$


where $m_{k}=\gamma_{k}+\left(1-\gamma_{k}\right)\left(\alpha_{k}+\beta_{k}\right)$. This means that $x^{*} \in C_{k}$. Hence

$$
\bigcap_{i=1}^{N} F\left(S_{i}\right) \cap \operatorname{Sol}(E P(f)) \subseteq C_{k}, \quad \forall k \geq 0 .
$$

Step 3. Now, we have to prove that

$$
\bigcap_{i=1}^{N} F\left(S_{i}\right) \cap \operatorname{Sol}(E P(f)) \subseteq C_{k} \cap Q_{k}
$$

for all $k \geq 0$.

We show this assertion by mathematical induction. For $k=0$ we have $Q_{0}=C$. Hence by Step 2, we obtain

$$
\bigcap_{i=1}^{N} F\left(S_{i}\right) \cap \operatorname{Sol}(E P(f)) \subseteq P_{0} \cap Q_{0}
$$

Assume that for some $k \geq 0$,

$$
\bigcap_{i=1}^{N} F\left(S_{i}\right) \cap \operatorname{Sol}(E P(f)) \subseteq C_{k} \cap Q_{k}
$$

From $x^{k+1}=\operatorname{Pr}_{C_{k} \cap Q_{k}}\left(x^{0}\right)$ it follows that

$$
\left\langle x^{k+1}-x, x^{0}-x^{k+1}\right\rangle \geq 0, \quad \forall x \in C_{k} \cap Q_{k} .
$$

Using this and (3.14), we have

$$
\left\langle x^{k+1}-x, x^{0}-x^{k+1}\right\rangle \geq 0, \quad \forall x \in \bigcap_{i=1}^{N} F\left(S_{i}\right) \cap \operatorname{Sol}(E P(f)) .
$$

Hence we have

$$
\bigcap_{i=1}^{N} F\left(S_{i}\right) \cap \operatorname{Sol}(E P(f)) \subseteq Q_{k+1}
$$

Then it follows from Step 2 that

$$
\bigcap_{i=1}^{N} F\left(S_{i}\right) \cap \operatorname{Sol}(E P(f)) \subseteq C_{k+1} \cap Q_{k+1} .
$$

Consequently, we have

$$
\bigcap_{i=1}^{N} F\left(S_{i}\right) \cap \operatorname{Sol}(E P(f)) \subseteq C_{k} \cap Q_{k}, \quad \forall k \geq 0 .
$$


Step 4. Next, we claim that

$$
\begin{aligned}
\lim _{k \rightarrow \infty}\left\|x^{k+1}-x^{k}\right\| & =\lim _{k \rightarrow \infty}\left\|x^{k}-z^{k}\right\| \\
& =\lim _{k \rightarrow \infty}\left\|x^{k}-y^{k}\right\| \\
& =\lim _{k \rightarrow \infty}\left\|x^{k}-t^{k}\right\| \\
& =\lim _{k \rightarrow \infty}\left\|\bar{S}_{k}\left(t^{k}\right)-t^{k}\right\| \\
& =0 .
\end{aligned}
$$

It follows from Step 2 and $x^{k+1}=\operatorname{Pr}_{C_{k} \cap Q_{k}}\left(x^{0}\right)$ that

$$
\left\|x^{k+1}-x^{0}\right\| \leq\left\|\operatorname{Pr}_{\bigcap_{i=1}^{N} F\left(S_{i}\right) \cap \operatorname{Sol}(E P(f))}\left(x^{0}\right)-x^{0}\right\|, \quad \forall k \geq 0 .
$$

Hence, we get that $\left\{x^{k}\right\}$ is bounded. By Step 1 , also the sequences $\left\{t^{k}\right\}$ and $\left\{z^{k}\right\}$ are bounded. Otherwise, we have

$$
\left\langle x^{k}-x, x^{0}-x^{k}\right\rangle \geq 0, \quad \forall x \in Q_{k},
$$

and hence $x^{k}=\operatorname{Pr}_{Q_{k}}\left(x^{0}\right)$. Using this and $x^{k+1} \in C_{k} \cap Q_{k} \subseteq Q_{k}$, we have

$$
\left\|x^{k}-x^{0}\right\| \leq\left\|x^{k+1}-x^{0}\right\|, \quad \forall k \geq 0 .
$$

Therefore, there exists

$$
A=\lim _{k \rightarrow \infty}\left\|x^{k}-x^{0}\right\|
$$

Using $x^{k}=\operatorname{Pr}_{Q_{k}}\left(x^{0}\right), x^{k+1} \in Q_{k}$ and the property of projections

$$
\left\|\operatorname{Pr}_{Q_{k}}(x)-x\right\|^{2} \leq\|x-y\|^{2}-\left\|\operatorname{Pr}_{Q_{k}}(x)-y\right\|^{2}, \quad \forall x \in H, y \in Q_{k},
$$

we have

$$
\left\|x^{k+1}-x^{k}\right\|^{2} \leq\left\|x^{k+1}-x^{0}\right\|^{2}-\left\|x^{k}-x^{0}\right\|^{2}, \quad \forall k \geq 0 .
$$

Combining this and (3.16), we get

$$
\lim _{k \rightarrow \infty}\left\|x^{k+1}-x^{k}\right\|=0
$$

It follows from $x^{k+1}=\operatorname{Pr}_{C_{k} \cap Q_{k}}\left(x^{0}\right)$ that $x^{k+1} \in C_{k}$, i.e.,

$$
\left\|z^{k}-x^{k+1}\right\| \leq\left\|x^{k}-x^{k+1}\right\|
$$

Hence

$$
\left\|x^{k}-z^{k}\right\| \leq\left\|x^{k}-x^{k+1}\right\|+\left\|x^{k+1}-z^{k}\right\| \leq 2\left\|x^{k}-x^{k+1}\right\|, \quad \forall k \geq 0 .
$$


Then, by (3.17), we have

$$
\lim _{k \rightarrow \infty}\left\|x^{k}-z^{k}\right\|=0
$$

Step 2 and (3.16) imply that $\left\{t^{k}\right\}$ is bounded, and hence $\left\{\bar{S}_{k}\left(t^{k}\right)-t^{k}\right\}$ and $\left\{z^{k}\right\}$ are also bounded.

By (3.13), we have

$$
\begin{aligned}
\beta_{k}\left(\frac{\alpha_{k}}{\alpha_{k}+\beta_{k}}-\bar{L}\right)\left\|\bar{S}_{k}\left(t^{k}\right)-t^{k}\right\|^{2} & \leq\left\|x^{k}-x^{*}\right\|^{2}-\left\|z^{k}-x^{*}\right\|^{2} \\
& =\left(\left\|x^{k}-x^{*}\right\|-\left\|z^{k}-x^{*}\right\|\right)\left(\left\|x^{k}-x^{*}\right\|+\left\|z^{k}-x^{*}\right\|\right) \\
& \leq\left\|x^{k}-z^{k}\right\|\left(\left\|x^{k}-x^{*}\right\|+\left\|z^{k}-x^{*}\right\|\right) .
\end{aligned}
$$

From this and (3.18), we obtain

$$
\lim _{k \rightarrow \infty}\left\|\bar{S}_{k}\left(t^{k}\right)-t^{k}\right\|=0
$$

Using (3.13), we also have

$$
m_{k}\left(1-2 \lambda_{k} c_{1}\right)\left\|x^{k}-y^{k}\right\|^{2} \leq\left\|x^{k}-z^{k}\right\|\left(\left\|x^{k}-x^{*}\right\|+\left\|z^{k}-x^{*}\right\|\right) \text {, }
$$

and hence

$$
\lim _{k \rightarrow \infty}\left\|x^{k}-y^{k}\right\|=0
$$

Similarly, we have

$$
\lim _{k \rightarrow \infty}\left\|t^{k}-y^{k}\right\|=0
$$

Combining (3.20), (3.21) and $\left\|x^{k}-t^{k}\right\| \leq\left\|x^{k}-y^{k}\right\|+\left\|y^{k}-t^{k}\right\|$, we have

$$
\lim _{k \rightarrow \infty}\left\|x^{k}-t^{k}\right\|=0
$$

This completes the proof of Step 4.

In Step 5 and Step 6 of this theorem, we consider weakly clusters of $\left\{x^{k}\right\}$. It follows from (3.15) that the sequence $\left\{x^{k}\right\}$ is bounded, and hence there exists a subsequence $\left\{x^{k_{j}}\right\}$ converging weakly to $\bar{x}$ as $j \rightarrow \infty$. By Step 4 , also the sequences $\left\{y^{k_{j}}\right\},\left\{t^{k_{j}}\right\}$ and $\left\{z^{k_{j}}\right\}$ converge weakly to $\bar{x}$.

Step 5. Claim that $\bar{x} \in \bigcap_{i=1}^{N} F\left(S_{i}\right)$.

For each $i=1, \ldots, N$, we suppose that $\left\{\lambda_{k_{j}, i}\right\}$ converges $\bar{\lambda}_{i}$ as $j \rightarrow \infty$ such that $\sum_{i=1}^{p} \bar{\lambda}_{i}=1$. Then we have

$$
S_{k_{j}}(x) \rightarrow S(x):=\sum_{i=1}^{N} \bar{\lambda}_{i} S_{i}(x) \quad(\text { as } j \rightarrow \infty), \forall x \in C .
$$


Since $\sum_{i=1}^{N} \bar{\lambda}_{i}=1$, from Step 4 and

$$
\begin{aligned}
\left\|t^{k_{j}}-S\left(t^{k_{j}}\right)\right\| & \leq\left\|t^{k_{j}}-\bar{S}_{k_{j}}\left(t^{k_{j}}\right)\right\|+\left\|\bar{S}_{k_{j}}\left(t^{k_{j}}\right)-S\left(t^{k_{j}}\right)\right\| \\
& =\left\|t^{k_{j}}-\bar{S}_{k_{j}}\left(t^{k_{j}}\right)\right\|+\left\|\sum_{i=1}^{N} \lambda_{k_{j}, i} S_{i}\left(t^{k_{j}}\right)-\sum_{i=1}^{N} \bar{\lambda}_{i} S_{i}\left(t^{k_{j}}\right)\right\| \\
& =\left\|t^{k_{j}}-\bar{S}_{k_{j}}\left(t^{k_{j}}\right)\right\|+\left\|\sum_{i=1}^{N}\left(\lambda_{k_{j}, i}-\bar{\lambda}_{i}\right) S_{i}\left(t^{k_{j}}\right)\right\| \\
& \leq\left\|t^{k_{j}}-\bar{S}_{k_{j}}\left(t^{k_{j}}\right)\right\|+\sum_{i=1}^{N}\left|\lambda_{k_{j}, i}-\bar{\lambda}_{i}\right|\left\|S_{i}\left(t^{k_{j}}\right)\right\|,
\end{aligned}
$$

we obtain that $\lim _{k \rightarrow \infty}\left\|t^{k_{j}}-S\left(t^{k_{j}}\right)\right\|=0$. By Proposition 2.3(b), we have

$$
\bar{x} \in F(S)=F\left(\sum_{i=1}^{N} \bar{\lambda}_{i} S_{i}\right) .
$$

Then, it implies that $\bar{x} \in \bigcap_{i=1}^{N} F\left(S_{i}\right)$ from Proposition 2.3(e).

Step 6. Now we prove that if $x^{k_{j}} \rightarrow \bar{x}$ as $j \rightarrow \infty$, then we have $\bar{x} \in \operatorname{Sol}(E P(f))$.

Since $y^{k}$ is the unique strongly convex problem

$$
\min \left\{\frac{1}{2}\left\|x-x^{k}\right\|^{2}+f\left(x^{k}, y\right) \mid y \in C\right\},
$$

from Lemma 3.1, we have

$$
0 \in \partial_{2}\left(\lambda_{k} f\left(x^{k}, y\right)+\frac{1}{2}\left\|y-x^{k}\right\|^{2}\right)\left(y^{k}\right)+N_{C}\left(y^{k}\right) .
$$

It follows that

$$
0=\lambda_{k} w+y^{k}-x^{k}+\bar{w}
$$

where $w \in \partial_{2} f\left(x^{k}, y^{k}\right)$ and $\bar{w} \in N_{C}\left(y^{k}\right)$. The definition of the normal cone $N_{C}$ implies that

$$
\left\langle y^{k}-x^{k}, y-y^{k}\right\rangle \geq \lambda_{k}\left\langle w, y^{k}-y\right\rangle, \quad \forall y \in C .
$$

On the other hand, since $f\left(x^{k}, \cdot\right)$ is subdifferentiable on $C$, by the Moreau-Rockafellar theorem [32], there exists $w \in \partial_{2} f\left(x^{k}, y^{k}\right)$ such that

$$
f\left(x^{k}, y\right)-f\left(x^{k}, y^{k}\right) \geq\left\langle w, y-y^{k}\right\rangle, \quad \forall y \in C
$$

Combining this with (3.23), we have

$$
\lambda_{k}\left(f\left(x^{k}, y\right)-f\left(x^{k}, y^{k}\right)\right) \geq\left\langle y^{k}-x^{k}, y^{k}-y\right\rangle, \quad \forall y \in C .
$$

Hence

$$
\lambda_{k_{j}}\left(f\left(x^{k_{j}}, y\right)-f\left(x^{k_{j}}, y^{k_{j}}\right)\right) \geq\left\langle y^{k_{j}}-x^{k_{j}}, y^{k_{j}}-y\right\rangle, \quad \forall y \in C .
$$


Then, using $\left\{\lambda_{k}\right\} \subset[a, b] \subset\left(0, \frac{1}{L}\right)$, Step $2, x^{k_{j}} \rightarrow \bar{x}$ as $j \rightarrow \infty$ and weak continuity of $f$, we have

$$
f(\bar{x}, y) \geq 0, \quad \forall y \in C .
$$

This means that $\bar{x} \in \operatorname{Sol}(E P(f))$.

Step 7. Finally, we claim that the sequences $\left\{x^{k}\right\},\left\{y^{k}\right\},\left\{z^{k}\right\}$ and $\left\{t^{k}\right\}$ converge strongly to the same point $x^{*}$, where

$$
x^{*}=\operatorname{Pr}_{\bigcap_{i=1}^{N} F\left(S_{i}\right) \cap \operatorname{Sol}(E P(f))}\left(x^{0}\right) .
$$

From Step 5 and Step 6 it follows that for every weakly cluster point $\bar{x}$ of the sequence $\left\{x^{k}\right\}$,

$$
\bar{x} \in \bigcap_{i=1}^{N} F\left(S_{i}\right) \cap \operatorname{Sol}(E P(f)) .
$$

On the other hand, using the definition of $Q_{k}$, we have

$$
x^{k}=\operatorname{Pr}_{Q_{k}}\left(x^{0}\right) .
$$

Combining this with (3.15), we obtain

$$
\left\|x^{0}-x^{k}\right\| \leq\left\|x^{0}-x\right\|
$$

for all $x \in \bigcap_{i=1}^{N} F\left(S_{i}, C\right) \cap \operatorname{Sol}(E P(f))$. For $x=x^{*}$, we have

$$
\left\|x^{0}-x^{k}\right\| \leq\left\|x^{0}-x^{*}\right\|
$$

By Lemma 3.2, we know that the sequence $\left\{x^{k}\right\}$ converges strongly to $x^{*}$ as $k \rightarrow \infty$, where

$$
x^{*}=\operatorname{Pr}_{\bigcap_{i=1}^{N} F\left(S_{i}, C\right) \cap \operatorname{Sol}(E P(f))}\left(x^{0}\right) .
$$

We also have that $y^{k}, z^{k}, t^{k} \rightarrow x^{*}$ as $k \rightarrow \infty$ by Step 4 .

\section{Applications}

Let $C$ be a nonempty closed convex subset of a real Hilbert space $H$. Let $F$ be a function from $C$ into $H$. In this section, we consider the variational inequality problem which is presented as follows:

Find $x^{*} \in C$ such that

$$
\left\langle F\left(x^{*}\right), x-x^{*}\right\rangle \geq 0, \quad \forall x \in C .
$$

Let $f: C \times C \rightarrow \mathbf{R}$ be defined by $f(x, y):=\langle F(x), y-x\rangle$. Then problem $E P(f)$ can be written in $V I(F)$. The set of solutions of $V I(F)$ is denoted by $\operatorname{Sol}(V I(F))$.

The function $F$ is called 
- strongly monotone on $C$ with $\beta>0$ if

$$
\langle F(x)-F(y), x-y\rangle \geq \beta\|x-y\|^{2}, \quad \forall x, y \in C ;
$$

- monotone on $C$ if

$$
\langle F(x)-F(y), x-y\rangle \geq 0, \quad \forall x, y \in C ;
$$

- pseudomonotone on $C$ if

$$
\langle F(y), x-y\rangle \geq 0 \Rightarrow\langle F(x), x-y\rangle \geq 0, \quad \forall x, y \in C
$$

- Lipschitz continuous on $C$ with constants $L>0$ if

$$
\|F(x)-F(y)\| \leq L\|x-y\|, \quad \forall x, y \in C
$$

Since

$$
\begin{aligned}
y^{k} & =\operatorname{argmin}\left\{\lambda_{k} f\left(x^{k}, y\right)+\frac{1}{2}\left\|y-x^{k}\right\|^{2} \mid y \in C\right\} \\
& =\operatorname{argmin}\left\{\lambda_{k}\left\langle F\left(x^{k}\right), y-x^{k}\right\rangle+\frac{1}{2}\left\|y-x^{k}\right\|^{2} \mid y \in C\right\} \\
& =\operatorname{Pr}_{C}\left(x^{k}-\lambda_{k} F\left(x^{k}\right)\right),
\end{aligned}
$$

from Algorithm 2.7, we obtain the algorithm for finding a common element of the set of fixed points of $p$ strict pseudocontractions and the solution set of variational inequality problem $V I(F)$.

\section{Algorithm 4.1}

Initialization: Choose positive sequences $\left\{\lambda_{k}\right\},\left\{\alpha_{k}\right\},\left\{\beta_{k}\right\},\left\{\gamma_{k}\right\}$ and $\left\{\lambda_{k, i}\right\}$ satisfying the conditions:

$$
\left\{\begin{array}{l}
\alpha_{k}+\beta_{k} \leq 1, \quad \forall k \geq 0, \\
\liminf _{k \rightarrow \infty} \beta_{k} \in(0,1), \\
\liminf _{k \rightarrow \infty} \frac{\alpha_{k}}{\alpha_{k}+\beta_{k}} \in(\bar{L}, 1), \quad \text { where } \bar{L}:=\max \left\{L_{i} \mid 1 \leq i \leq N\right\} \\
\liminf _{k \rightarrow \infty}\left(\gamma_{k}+\left(1-\gamma_{k}\right)\left(\alpha_{k}+\beta_{k}\right)\right)>0, \quad\left\{\gamma_{k}\right\} \subset(0,1), \\
\left\{\lambda_{k}\right\} \subset[a, b] \quad \text { for some } a, b \in\left(0, \frac{1}{L}\right), \\
\sum_{i=1}^{N} \lambda_{k, i}=1 \quad \text { for all } k \geq 1 .
\end{array}\right.
$$

Find an initial point $x^{0} \in C$.

Iteration $k$ : Perform the three steps below.

- Step 1. Solve two strongly convex programs:

$$
\left\{\begin{array}{l}
y^{k}:=\operatorname{Pr}_{C}\left(x^{k}-\lambda_{k} F\left(x^{k}\right)\right), \\
t^{k}:=\operatorname{Pr}_{C}\left(x^{k}-\lambda_{k} F\left(y^{k}\right)\right) .
\end{array}\right.
$$


- Step 2. Compute the iterations

$$
\left\{\begin{array}{l}
\bar{y}^{k}:=\left(1-\gamma_{k}\right) x^{k}+\gamma_{k} t^{k} \\
z^{k}:=\left(1-\alpha_{k}-\beta_{k}\right) \bar{y}^{k}+\alpha_{k} t^{k}+\beta_{k} \sum_{i=1}^{N} \lambda_{k, i} S_{i}\left(t^{k}\right)
\end{array}\right.
$$

- Step 3. Set

$$
\left\{\begin{array}{l}
C_{k}:=\left\{z \in C \mid\left\|z^{k}-z\right\|^{2} \leq\left\|x^{k}-z\right\|^{2}-\beta_{k}\left(\frac{\alpha_{k}}{\alpha_{k}+\beta_{k}}-\bar{L}\right)\left\|\bar{S}_{k}\left(t^{k}\right)-t^{k}\right\|^{2}\right\}, \\
Q_{k}:=\left\{z \in C \mid\left\langle x^{k}-z, x^{0}-x^{k}\right\rangle \geq 0\right\} .
\end{array}\right.
$$

Compute $x^{k+1}:=\operatorname{Pr}_{C_{k} \cap Q_{k}}\left(x^{0}\right)$.

Increase $k$ by one and go back to Step 1 .

Now, we can prove the following convergence theorem with respect to $V I(F)$ from Theorem 3.3.

Theorem 4.2 Let $C$ be a nonempty closed convex subset of a real Hilbert space H. Let $F$ be a function from $C$ into $H$ such that $F$ is pseudomonotone, weakly continuous and $L$-Lipschitz continuous on $C$. If each $i=1, \ldots, N, S_{i}: C \rightarrow C$ is $L_{i}$-strict pseudocontraction for some $0 \leq L_{i}<1$ and

$$
\bigcap_{i=1}^{N} F\left(S_{i}\right) \cap \operatorname{Sol}(V I(F)) \neq \emptyset,
$$

then the sequences $\left\{x^{k}\right\},\left\{y^{k}\right\}$ and $\left\{z^{k}\right\}$ generated by Algorithm 4.1 converge strongly to the same point $x^{*} \in \bigcap_{i=1}^{p} \operatorname{Fix}\left(S_{i}\right) \cap \operatorname{Sol}(F, C)$, where

$$
x^{*}=\operatorname{Pr}_{\bigcap_{i=1}^{N} F\left(S_{i}\right) \cap \operatorname{Sol}(V I(F))}\left(x^{0}\right) .
$$

\section{Competing interests}

The authors declare that they have no competing interests.

\section{Authors' contributions}

The main idea of this paper was proposed by JKK. JKK and WHL prepared the manuscript initially and performed all the steps of proof in this research. Both authors read and approved the final manuscript.

\section{Acknowledgements}

This work was supported by the Kyungnam University Foundation Grant 2011.

Received: 28 November 2012 Accepted: 8 March 2013 Published: 26 March 2013

\section{References}

1. Blum, E, Oettli, W: From optimization and variational inequality to equilibrium problems. Math. Stud. 63, 127-149 (1994)

2. Daniele, P. Giannessi, F, Maugeri, A: Equilibrium Problems and Variational Models. Kluwer Academic, Dordrecht (2003)

3. Anh, PN: A logarithmic quadratic regularization method for solving pseudo-monotone equilibrium problems. Acta Math. Vietnam. 34, 183-200 (2009)

4. Anh, PN: An LQP regularization method for equilibrium problems on polyhedral. Vietnam J. Math. 36, 209-228 (2008)

5. Chang, SS, Cho, YJ, Kim, JK: Approximation methods of solutions for equilibrium problem in Hilbert spaces. Dyn. Syst. Appl. 17, 503-508 (2008)

6. Mastroeni, G: Gap function for equilibrium problems. J. Glob. Optim. 27, 411-426 (2004)

7. Peng, JW: Iterative algorithms for mixed equilibrium problems, strict pseudocontractions and monotone mappings. J. Optim. Theory Appl. 144, 107-119 (2010) 
8. Goebel, K, Kirk, WA: Topics on Metric Fixed Point Theory. Cambridge University Press, Cambridge (1990)

9. Kim, JK, Sahu, DR, Nam, YM: Convergence theorem for fixed points of nearly uniformly L-Lipschitzian asymptotically generalized $\phi$-hemicontractive mappings. Nonlinear Anal. TMA 71, 2833-2838 (2009). doi:10.1016/j.na.2009.06.091

10. Kim, JK, Nam, YM, Sim, JY: Convergence theorem of implicit iterative sequences for a finite family of asymptotically quasi-nonexpansive type mappings. Nonlinear Anal. TMA 71, 2839-2848 (2009). doi:10.1016/j.na.2009.06.090

11. Chang, SS, Lee, HWJ, Chan, CK, Kim, JK: Approximating solutions of variational inequalities for asymptotically nonexpansive mappings. Appl. Math. Comput. 212, 51-59 (2009)

12. Kim, JK: Strong convergence theorems by hybrid projection methods for equilibrium problems and fixed point problems of the asymptotically quasi- $\boldsymbol{\phi}$-nonexpansive mappings. Fixed Point Theory Appl. (2011). doi:10.1186/1687-1812-2011-10

13. Kim, JK, Cho, SY, Qin, X: Some results on generalized equilibrium problems involving strictly pseudocontractive mappings. Acta Math. Sci., Ser. B 31(5), 985-996 (2011)

14. Nadezhkina, N, Takahashi, W: Weak convergence theorem by an extragradient method for nonexpansive mappings and monotone mappings. J. Optim. Theory Appl. 128, 191-201 (2006)

15. Takahashi, S, Takahashi, W: Viscosity approximation methods for equilibrium problems and fixed point problems in Hilbert spaces. J. Math. Anal. Appl. 331, 506-515 (2007)

16. Takahashi, S, Toyoda, M: Weakly convergence theorems for nonexpansive mappings and monotone mappings. J. Optim. Theory Appl. 118, 417-428 (2003)

17. Zeng, LC, Yao, JC: Strong convergence theorem by an extragradient method for fixed point problems and variational inequality problems. Taiwan. J. Math. 10, 1293-1303 (2010)

18. Li, XS, Huang, J, Kim, JK: General viscosity approximation methods for common fixed points of nonexpansive semigroup in Hilbert spaces. Fixed Point Theory Appl. 2011, Article ID 783502 (2011). doi:10.1155/2011/783502

19. Li, XS, Kim, JK, Huang, NJ: Viscosity approximation of common fixed points for L-Lipschitzian semigroup of pseudocontractive mappings in Banach spaces. J. Inequal. Appl. 2009, Article ID 936121 (2009). doi:10.1155/2009/936121

20. Chen, R, Shen, X, Cui, S: Weak and strong convergence theorems for equilibrium problems and countable strict pseudocontractions mappings in Hilbert space. J. Inequal. Appl. (2010). doi:10.1155/2010/474813

21. Kim, JK, Buong, N: Regularization inertial proximal point algorithm for monotone hemicontinuous mapping and inverse strongly monotone mappings in Hilbert spaces. J. Inequal. Appl. 2010, Article ID 451916 (2010). doi:10.1155/2010/451916

22. Kim, JK, Anh, PN, Nam, YM: Strong convergence of an extended extragradient method for equilibrium problems and fixed point problems. J. Korean Math. Soc. 47, 187-200 (2011)

23. Kim, JK, Tuyen, TM: Regularization proximal point algorithm for finding a common fixed point of a finite family of nonexpansive mappings in Banach spaces. Fixed Point Theory Appl. 2011, Article ID 52 (2011). doi:10.1186/1687-1812-2011-52

24. Schaible, S, Karamardian, S, Crouzeix, JP: Characterizations of generalized monotone maps. J. Optim. Theory Appl. 76, 399-413 (1993)

25. Acedo, GL, Xu, HK: Iterative methods for strict pseudo-contractions in Hilbert spaces. Nonlinear Anal. 67, 2258-2271 (2007)

26. Marino-Yanes, C, Xu, HK: Strong convergence of the CQ method for fixed point processes. Nonlinear Anal. 64 2400-2411 (2006)

27. Ceng, LC, Petrusel, A, Lee, C, Wong, MM: Two extragradient approximation methods for variational inequalities and fixed point problems of strict pseudo-contractions. Taiwan. J. Math. 13, 607-632 (2009)

28. Wang, S, Cho, YJ, Qin, X: A new iterative method for solving equilibrium problems and fixed point problems for infinite family of nonexpansive mappings. Fixed Point Theory Appl. (2010). doi:10.1155/2010/165098

29. Wang, S, Guo, B: New iterative scheme with nonexpansive mappings for equilibrium problems and variational inequality problems in Hilbert spaces. J. Comput. Appl. Math. 233, 2620-2630 (2010)

30. Yao, Y, Liou, YC, Wu, YJ: An extragradient method for mixed equilibrium problems and fixed point problems. Fixed Point Theory Appl. (2009). doi:10.1155/2009/632819

31. Rockafellar, RT: Convex Analysis. Princeton University Press, Princeton (1970)

32. Kim, JK, Cho, SY, Qin, X: Hybrid projection algorithms for generalized equilibrium problems and strictly pseudocontractive mappings. J. Inequal. Appl. 2010, Article ID 312602 (2010). doi:10.1155/2010/312602

doi:10.1186/1029-242X-2013-128

Cite this article as: Kim and Lim: A new iterative algorithm of pseudomonotone mappings for equilibrium problems

in Hilbert spaces. Journal of Inequalities and Applications 2013 2013:128. 